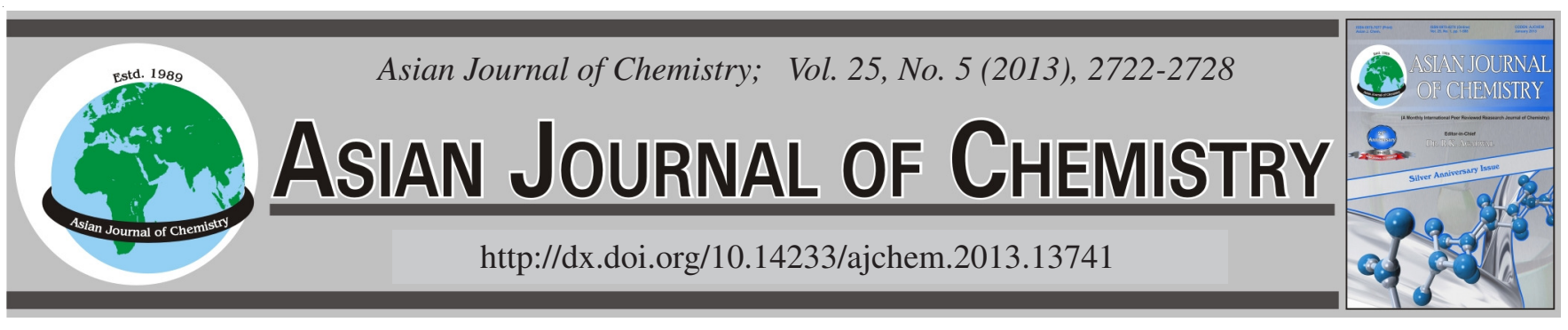

\title{
Supported Ionic Liquids on Solid Materials as Catalysts for the Synthesis of Propylene Glycol Methyl Ether
}

\author{
Minh NGoc HaA ${ }^{1}$, Roger Whiting ${ }^{2}$, Sheng $\mathrm{HAN}^{1}$ and Yuhong Wang ${ }^{1, *}$
}

${ }^{1}$ Research Institute of Applied Catalysis, School of Chemical and Enviormental Engineering, Shanghai Institute of Technology, Shanghai 201418, P.R. China

${ }^{2}$ School of Applied Science, Faculty of Health and Environmental Sciences, Auckland University of Technology, Auckland, New Zealand

*Corresponding author: Tel: +86 21 60873558; E-mail: yuhong_wang502@ hotmail.com

\section{INTRODUCTION}

Ionic liquids (ILs), which are organic salts with a melting point below $100{ }^{\circ} \mathrm{C}$, have attracted much attention in recent years. Compared with traditional organic solvents, ionic liquids have a negligible vapor pressure, high thermal stability and are non-flammable and reusable ${ }^{1-3}$. They have been investigated extensively in organic synthesis as solvents or catalysts ${ }^{1,3-10}$. As both the anionic and the cationic part of ionic liquids can be easily varied, their properties can be tuned for specific purposes. In recent years, a great number of functional ionic liquids have been synthesized for different purposes ${ }^{11-14}$.

Recently, the immobilization of ionic liquids onto solid supports and the preparation of catalytic materials assisted by ionic liquids have attracted significant attention. Several kinds of supports, including silica gel, polymers, montmorillonite, etc., have been used to immobilize ionic liquids via different interactions. Natural clays are environmentally friendly materials with crystalline structures and relatively large surface areas which can be used as catalyst supports. Attapulgite (Atta) is a crystalline hydrated magnesium aluminum silicate with a unique three-dimensional structure. It has a fibrous morphology with exchangeable cations and reactive - $\mathrm{OH}$ groups on its surface. Attapulgite has the structural formula $\mathrm{Si}_{8} \mathrm{O}_{20} \mathrm{Mg}_{5}(\mathrm{Al})(\mathrm{OH})_{2}\left(\mathrm{H}_{2} \mathrm{O}\right)_{4} \cdot 4 \mathrm{H}_{2} \mathrm{O}$ and its structure was studied by Bradley in 1940. Because of its structural morphology and high surface area, attapulgite has received considerable attention with regard to the adsorption and the removal of heavy-metal ions. Mesoporous $\mathrm{SiO}_{2}$ based minerals are attractive supports due to properties, such as excellent chemical and thermal stability, high porosity, large surface area and high surface concentration of silanols. MCM-41 has a large void due to the presence of the mesopores and concomitantly a rather low density. This property makes MCM-41 very interesting as a support material for heterogeneous catalysts. Moreover, because MCM-41 exclusively contains mesopores it can provide both access to large molecules and alleviate diffusion problems, which are frequently encountered in microporous materials such as zeolites. Three support materials were selected for this study based on their structural morphology and high surface areas.

Propylene glycol methyl ether (PGME) is widely used as solvent because of its ether bond and hydroxyl group, which are hydrophobic and hydrophilic, respectively. It is considered to be a safe substitute for toxic ethylene glycol ether because of the negligible toxicity of propylene glycol ether. Propylene glycol methyl ether is conventionally derived from addition of methanol to propylene oxide (PO) catalyzed by acid or base.

Propylene glycol methyl ether is produced by the reaction of propylene oxide with methanol using a catalyst as shown below.

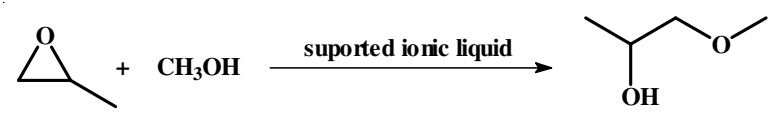


Generally, the mechanism is considered to be as follows ${ }^{15}$. Basic catalysis:

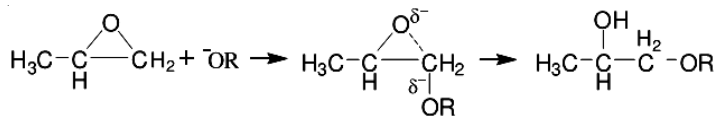

Acidic catalysis:

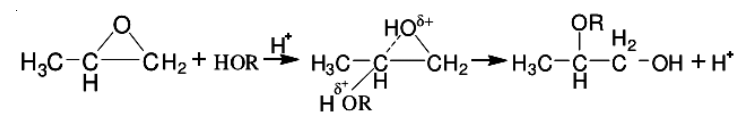

The epoxide ring of propylene oxide can open at either of the C-O bonds, to form primary or secondary alcohols. Compared to secondary alcohols, the primary alcohols (such as 2-methoxy-1-propanol) exhibit reproductive and developmental toxicity ${ }^{16}$. Therefore, a high selectivity to secondary alcohols is required for this process. An acid catalyst provides a mixture of secondary and primary alcohols and the proportion of the isomers depends on the acid strength. However, the ring of propylene oxide might preferentially open at the least sterically hindered position over a basic catalyst, leading to mostly the secondary alcohol 1-methoxy-2-propanol. Therefore the base catalysts are attracting much attention in the research and application of these materials.

In the past decades, a lot of homogenous base catalysts were exploited and showed high selectivity to secondary alcohols, but they had the drawbacks of separation, liquid waste treatment and corrosion problems. Therefore solid bases were developed to catalyze the synthesis of propylene glycol ether. Those heterogeneous catalysts included anionic double hydroxide clays ${ }^{17}$, basic zeolites ${ }^{18}$ and basic metal oxides ${ }^{19,20}$.

In this work, ionic liquid 1,1,3,3-tetramethylguanidium lactate ([TMG][Lac]) was immobilized on different solid supports, i.e., MCM-41, Silica-gel and attapulgite. The as-prepared materials were used as the basic heterogeneous catalysts for preparing propylene glycol methyl ether from methanol and propylene oxide. Good yields and isomer selectivities were obtained over the three types of catalysts. The catalyses can be separated from the products easily by centrifugation and reused without a decrease in activity or selectivity.

\section{EXPERIMENTAL}

1,1,3,3-Tetramethylguanidine was purchased from Baigui Chemical Company (Shijiazhuang, China). Lactic acid was procured from Shanghai Jingchun Reagent Plant (Shanghai, China). Acetic acid, ethanol, methanol and propylene oxide, $\mathrm{C}_{16} \mathrm{H}_{33} \mathrm{~N}\left(\mathrm{CH}_{3}\right)_{3} \mathrm{Br}$, sodium silicate $\left(\mathrm{SiO}_{2} / \mathrm{Na}_{2} \mathrm{O}=3\right), \mathrm{CH}_{3} \mathrm{COOH}$, $\mathrm{NH}_{3} 25 \%$, tetraethoxyorthosilicate (TEOS) were from Beijing Chemical Plants (Beijing, China).

Attapulgite (Atta) provided by Hongfei Company (Jiangsu, China), the composition of the attapulgite clay was $61.23 \% \mathrm{SiO}_{2}, 11.42 \% \mathrm{Al}_{2} \mathrm{O}_{3}, 9.07 \% \mathrm{MgO}, 0.29 \% \mathrm{Na}_{2} \mathrm{O}$, $1.32 \% \mathrm{~K}_{2} \mathrm{O}, 0.34 \% \mathrm{CaO}, 7.2 \% \mathrm{Fe}_{2} \mathrm{O}_{3}, 1.07 \% \mathrm{TiO}_{2}, 0.023 \%$ $\mathrm{P}_{2} \mathrm{O}_{5}$ and $0.17 \% \mathrm{FeO}$. Other reagents and solvents were analytical grade and produced by Shanghai Chemical Reagents Company. All the chemicals were used as received.

\section{Preparation of the catalysts}

Preparation of [TMG][Lac] ionic liquid: [TMG][Lac] ionic liquid was prepared directly by neutralization of 1,1,3,3tetramethylguanidine with lactic acid at room temperature ${ }^{12}$.
Preparation of TMG/MCM-41: The MCM-41 material was directly prepared by sol-gel method ${ }^{13} .10 \mathrm{~g}$ of MCM-41 was dispersed in $150 \mathrm{~mL}$ of aqueous solution containing $5.3 \mathrm{~g}$ of [TMG][Lac] and stirred for $c a$. $6 \mathrm{~h}$. Then the MCM-41 was separated by filtration and treated with fresh [TMG][Lac] aqueous solution again. The treated MCM-41 was filtered, washed with a large amount of deionized water, dried at $105^{\circ} \mathrm{C}$ for $24 \mathrm{~h}$ and named as TMG/MCM-41.

Preparation of TMG/Silica-gel: A mixture of tetraethoxyorthosilicate (TEOS, $10 \mathrm{~mL})$ and $\mathrm{EtOH}(7 \mathrm{~mL})$ was heated to $60{ }^{\circ} \mathrm{C}$ and then ionic liquids containing (4 g) of [TMG][Lac] were immediately transferred into the TEOS. After the formation of a clear and homogeneous liquid mixture, hydrochloric acid ( $5 \mathrm{M}, 5 \mathrm{~mL}$ ) was added and the mixture gradually coagulated. After aging at $60{ }^{\circ} \mathrm{C}$ for $12 \mathrm{~h}$, the resultant solid material was dried in vacuum at $150^{\circ} \mathrm{C}$ for $3 \mathrm{~h}$ and $7 \mathrm{~g}$ of solid sample was obtained and named TMG/silica-gel.

It is worth noting that a dry solid sample of silica-gelconfined ionic liquid was obtained even with ionic liquid loading of up to $53 \mathrm{wt} \%$, indicating that the ionic liquid added could be completely encapsulated in the sol-gel. Therefore, the loadings of confined ionic liquids could be calculated according to the weight of ionic liquids added originally.

Preparation of TMG/Atta: Attapulgite was treated with $36.5 \%$ hydrochloric acid for $24 \mathrm{~h}$, followed by washing with distilled water until the washing water was $\mathrm{pH} 6.5$ then drying in vacuum at $50{ }^{\circ} \mathrm{C}$ for $24 \mathrm{~h}^{14}$. A $10.0 \mathrm{~g}$ sample of the purified attapulgite was dispersed in a $150 \mathrm{~mL}$ aqueous solution containing $5.3 \mathrm{~g}$ of [TMG][Lac] with stirring for $c a .6 \mathrm{~h}$. Then the attapulgite was separated via centrifugation and treated with fresh ionic liquid aqueous solution again. This procedure was repeated for a third time. The attapulgite (Atta) treated with aqueous solution of ionic liquid was filtered, washed with a large amount of deionized water, dried at $105^{\circ} \mathrm{C}$ for $24 \mathrm{~h}$ and named as TMG/Atta.

Characterization: FT-IR spectra of the samples as $\mathrm{KBr}$ discs were taken in the range of $4000-400 \mathrm{~cm}^{-1}$ on Nicolet360 FT-IR spectrometer. Thermal analysis experiments were performed using a TGA apparatus operated in the conventional TGA mode (SDT Q-600, TA Instrument) at the heating rate of $10 \mathrm{~K} / \mathrm{min}$ in a nitrogen atmosphere and the sample size depended on each type of catalyst. The specific surface area, total pore volume and average pore diameter were measured by $\mathrm{N}_{2}$ adsorption-desorption method using Micromeritics ASAP 2020 instrument. The samples were treated at $453 \mathrm{~K}$ for $3 \mathrm{~h}$ under vacuum and then the adsorption-desorption was conducted by passing nitrogen into the sample, which was kept under liquid.

Catalytic performance: The catalytic performance of the catalysts was evaluated in a $10 \mathrm{~mL}$ batch reactor with molar ratio of methanol to propylene oxide ranging from 1:1 to 5:1. After running at $323-373 \mathrm{~K}$ for the required time under magnetic stirring, the reactor was cooled down to room temperature. After centrifugal separation from the catalyst, the products were analyzed by GC (Fuli 9730) equipped with a FID detector and a capillary column (PEG20M: length $30 \mathrm{~m}$ $\times$ inner diameter $0.25 \mathrm{~mm} \times$ film thickness $0.25 \mu \mathrm{m}$ ). 1-Propanol was used as the internal standard to calculate the amount of products. The yield of propylene glycol methyl ether (primary 
and second alcohols) is defined as the ratio of number of moles of propylene glycol methyl ether produced in the reaction to the total moles of propylene oxide initially added. Selectivity to 1-methoxy-2-propanol is defined as the ratio of number of moles of 1-methoxy-2-propanol to the number of moles of the two isomers.

Recycling studies: After the first run, the catalyst was filtered, washed with three times distilled water, dried in an air-oven and then activated at $378 \mathrm{~K}$ for $24 \mathrm{~h}$ and used for the subsequent recycling studies.

\section{RESULTS AND DISCUSSION}

Characterization results: Before testing the catalytic activity of the catalysts, TMG/MCM-41, TMG/silica-gel and TMG/Atta were characterized with FT-IR. The existence of the $[\mathrm{TMG}]^{+}$was supported by the FT-IR analysis (Fig. 1). The specific peaks of TMG (TMG/MCM-41: $3548 v(\mathrm{~N}-\mathrm{H}), 3013$ $v(\mathrm{C}-\mathrm{H}), 1678 \mathrm{v}(\mathrm{C}=\mathrm{N}), 1597 \delta(\mathrm{N}-\mathrm{H}), 1398,1410 \delta(\mathrm{C}-\mathrm{H}) \mathrm{cm}^{-1}$; TMG/Silica-gel: $3385 v(\mathrm{~N}-\mathrm{H}), 2897 v(\mathrm{C}-\mathrm{H}), 1614 \mathrm{v}(\mathrm{C}=\mathrm{N})$, $1711,1623 \delta(\mathrm{N}-\mathrm{H}), 1476,1429 \mathrm{~cm}^{-1} \delta(\mathrm{C}-\mathrm{H})$; TMG/Atta: 3122 , $3598 v(\mathrm{~N}-\mathrm{H}), 2986 v(\mathrm{C}-\mathrm{H}), 1627 \mathrm{v}(\mathrm{C}=\mathrm{N}), 1543 \delta(\mathrm{N}-\mathrm{H}), 1491$, $\left.1412 \delta(\mathrm{C}-\mathrm{H}) \mathrm{cm}^{-1}\right)$ can be observed in the spectra of the three catalysts.

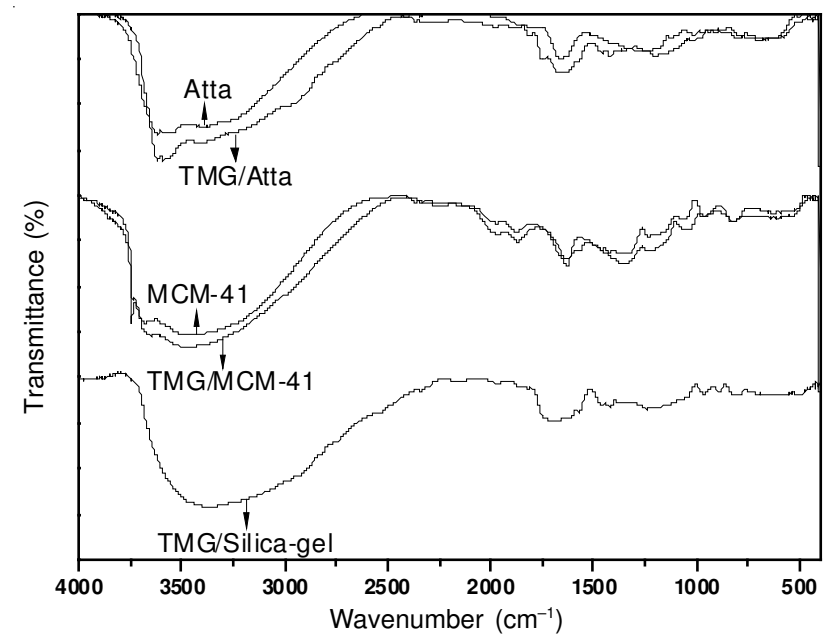

Fig. 1. FT-IR spectra of catalysts TMG/MCM-41, TMG/silica-gel and TMG/Atta

Fig. 2 is the $\mathrm{N}_{2}$ adsorption-desorption isotherm of TMG/ MCM-41, TMG/silica-gel and TMG/Atta. As presented in Fig. 2, the sample TMG/MCM-41 exhibited complementary textural-and framework-confined mesoporosity, as evidenced by the presence of two separate, well-expressed hysteresis loops. The position of the inflection in the $\mathrm{P} / \mathrm{P}_{0}=0.25-0.45$ region depends on the diameter of the mesopores and its sharpness indicates the uniformity of the narrow pore size distribution. It can also be seen in Fig. 2 that the point of inflection (TMG/ MCM-41 sample) shifts toward higher relative pressure $\left(\mathrm{P} / \mathrm{P}_{0}\right)$, which indicates an increase in the pore size suggesting the incorporation of 1,1,3,3-tetramethylguanidium. The analysis results showed that TMG/MCM-41 catalyst has a largest surface area of $777 \mathrm{~m}^{2} / \mathrm{g}$ due to its mesoporous structure. Because of the amorphous structure of TMG/Silica-gel and layered structure of TMG/Atta, they have a smaller surface area, respectively, as follows 219 and $110 \mathrm{~m}^{2} / \mathrm{g}$.
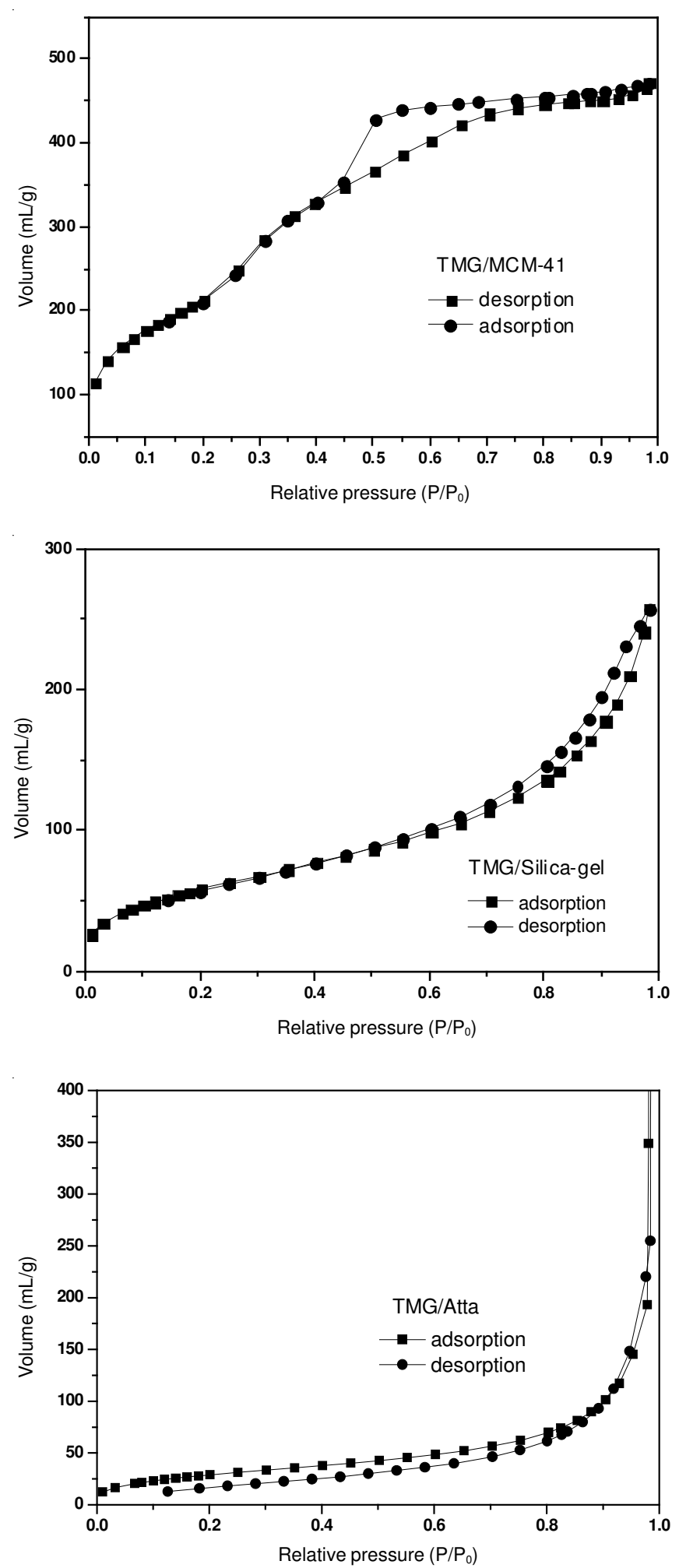

Fig. 2. $\mathrm{N}_{2}$ adsorption-desorption isotherms of TMG/MCM-41, TMG/silicagel and TMG/Atta

The thermal stability of the three catalysts was determined using thermogravimetric analysis. The weight loss curves for TMG/MCM-41, TMG/Silica-gel and TMG/Atta are presented in Fig. 3. In the TGA curve for TMG/MCM-41 sample, the weight losse is negligible due to a calcined MCM-41 used as starting material for preparation of impregnated catalysts. Therefore, in the current preparation method, the weight loss unchanged from the temperature of $100-800{ }^{\circ} \mathrm{C}$. The TGA 


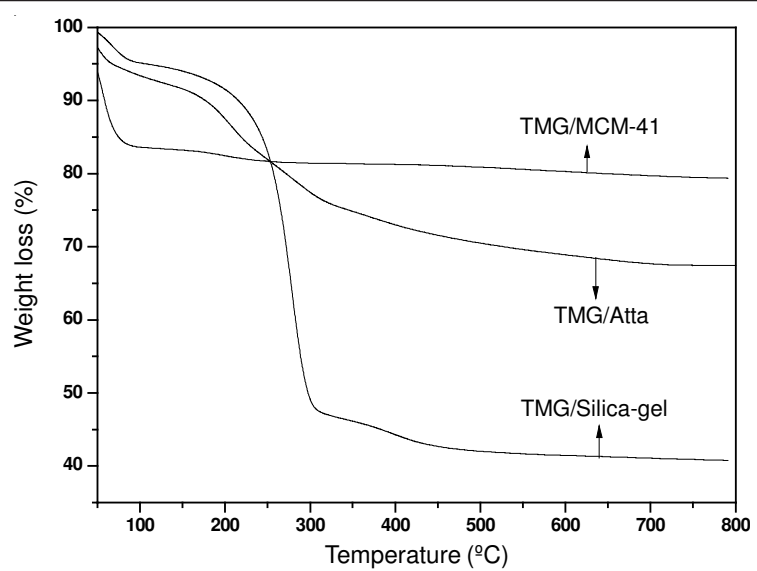

Fig. 3. TGA profiles of TMG/MCM-41, TMG/Silica-gel and TMG/Atta

profile for TMG/Silica-gel showed that most of the surfactant (TEOS) was decomposed in the temperature range of 100$300{ }^{\circ} \mathrm{C}$ and then the weight loss occurred at temperature below $300^{\circ} \mathrm{C}$. Thermogravimetric curve of TMG/Atta showed the weight losses were around $200-600^{\circ} \mathrm{C}$ likely attributed to the slower decomposition of $\left[\mathrm{SiO}_{4}\right]^{4-}$. The possible reason for the slower decomposion is that the interaction between TMG and $\left[\mathrm{SiO}_{4}\right]^{4-}$ is stronger than that between TMG and TEOS.

Catalytic activity of catalysts in the reaction of propylene oxide and methanol: The effect of the amount of the catalysts on the yield of propylene glycol methyl ether and the isomer selectivity to 1-methoxy-2-propanol is shown in Fig. 4. The propylene glycol methyl ether yield increases with the amount of catalysts; while the yield arrives at the maximum when the amount of TMG/MCM-41 is $0.25 \mathrm{~g}$. The isomer selectivity does not change with the amount of catalysts over three catalysts, indicating that the supported TMG catalysts are effective for producing 1-methoxy-2-propanol isomer.

Fig. 5 shows the influence of molar ratio of methanol to propylene oxide (MeOH/PO) on the yield of propylene glycol methyl ether and the isomer selectivity to 1-methoxy-2-propanol at $353 \mathrm{~K}$ for $3 \mathrm{~h}$. With the increase of the molar ratio of $\mathrm{MeOH} /$ $\mathrm{PO}$, propylene oxide could completely react with $\mathrm{MeOH}$ and the yield of propylene glycol methyl ether rapidly reaches a maximum value. When the molar ratio exceeds $3: 1$, the yield decreases slightly. The selectivity to 1-methoxy-2-propanol almost keeps constant over the three catalysts when changing the molar ratio of $\mathrm{MeOH} /$ propylene oxide.

As shown in Fig. 6, the dependence of the catalytic activity on the reaction temperature is different over the three catalysts. When the reaction temperature increases from 323 to $353 \mathrm{~K}$, the yield of reaction increased significantly (60-90\% for TMG/ MCM-41). Above $353 \mathrm{~K}$ the, the yield does not change with the increasing temperature. However the situation was different for each type of catalyst. The difference in the structure of three catalysts affects the yield differently at different reaction temperatures. The surface area of TMG/MCM-41 is the largest so TMG/MCM-41 shows greater catalytic activity than the other two catalysts at low temperatures. At high temperatures, the effect of reaction temperature exceeds the effect of surface area and so reaction of the three catalysts is similar. In addition, the effect of temperature on the selectivity is not obvious over the range of temperature for all three catalysts.
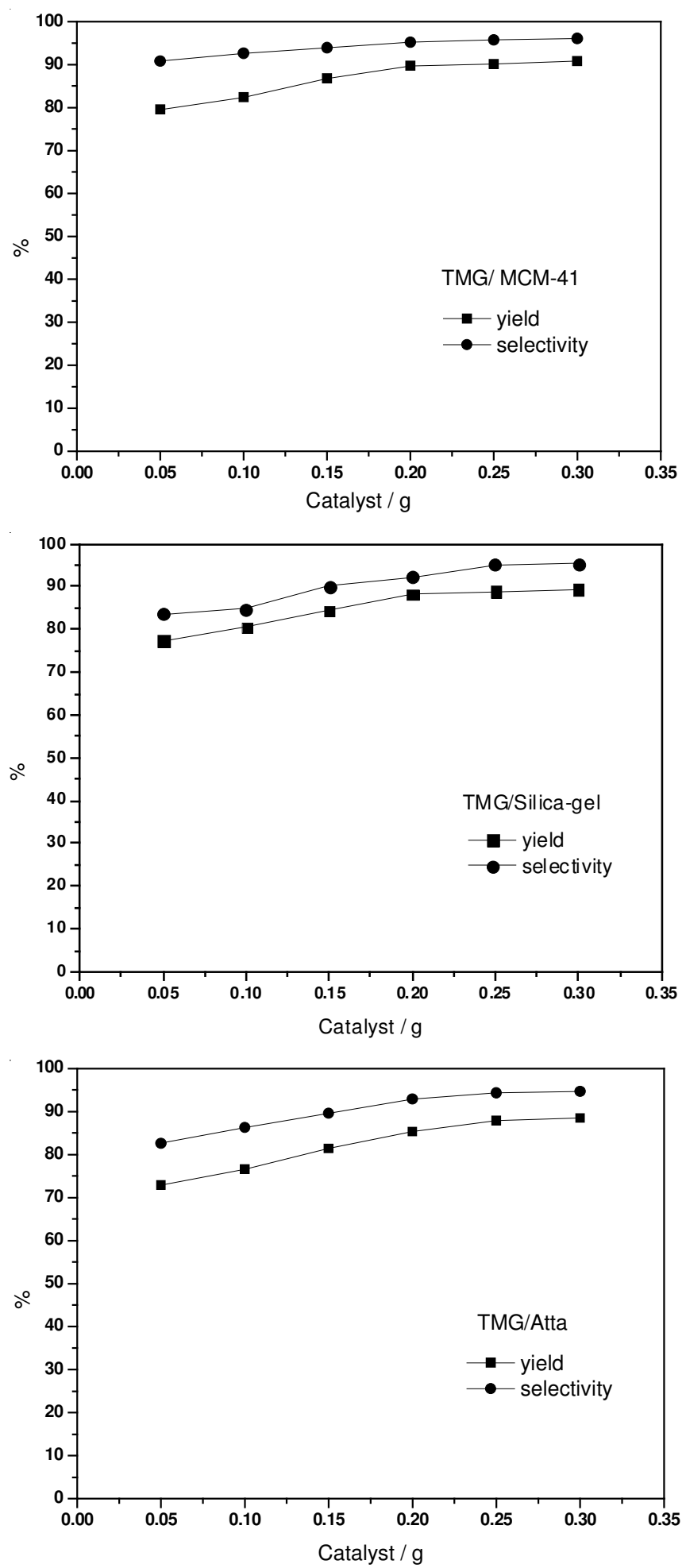

Fig. 4. Effect of the amount of the catalyst on the yield of propylene glycol methyl ether and isomer selectivity. Reaction conditions: PO, 0.1 M; $\mathrm{MeOH} / \mathrm{PO}$ molar ratio, 3 ; reaction temperature, $353 \mathrm{~K}$; reaction time, $3 \mathrm{~h}$

The dependence of the yields of propylene glycol methyl ether and the selectivities to 1-methoxy-2-propanol on the reaction time is presented in Fig. 7. In a relatively short reaction time, the conversion of propylene oxide is incomplete and the propylene glycol methyl ether yield is low. It can be seen that the maximum yield could be achieved at $3 \mathrm{~h}$ and no further increase in the yields of propylene glycol methyl ether is 

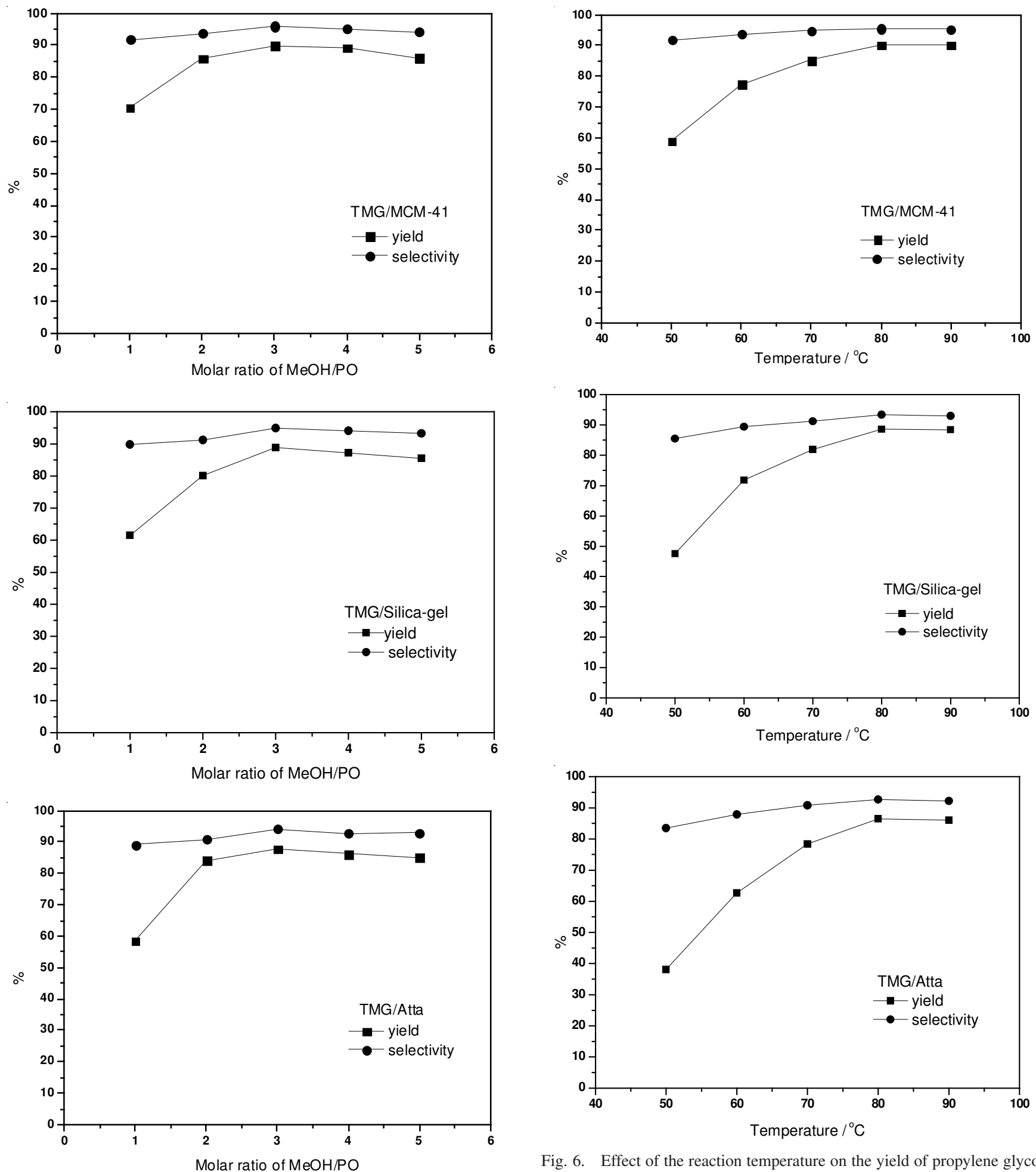

Fig. 5. Effect of molar ratio of $\mathrm{MeOH} / \mathrm{PO}$ on the yield of propylene glycol methyl ether and the isomer selectivity. Reaction conditions: PO, $0.1 \mathrm{M}$; catalyst, $0.25 \mathrm{~g}$; reaction temperature, $353 \mathrm{~K}$; reaction time, $3 \mathrm{~h}$

observed with prolonged reaction time over the three catalysts. But the yield over TMG/MCM-41 is higher than that over TMG/silica-gel and TMG/Atta when the reaction time was $1 \mathrm{~h}$. This might be attributed to the enormous difference of the surface area of the three supports. The high surface area favours the contact of reactants on the catalyst surface. This effect is especially obvious at short reaction time.

Fig. 6. Effect of the reaction temperature on the yield of propylene glycol methyl ether and the isomer selectivity. Reaction conditions: PO, $0.1 \mathrm{M} ; \mathrm{MeOH} / \mathrm{PO}$ molar ratio, 3 ; catalyst, $0.25 \mathrm{~g}$; reaction time, $3 \mathrm{~h}$

The reusability of TMG/MCM-41, TMG/Silica-gel and TMG/Atta were examined at the optimized reaction conditions and the results are shown in Fig. 8. The yield of propylene glycol methyl ether and the selectivity to 1-methoxy-2-propanol do not decrease after six recyclings, indicating that all catalysts are recyclable. The reduction in yield of propylene glycol methyl ether and the isomer selectivity after six cycles is negligible. For the three catalysts, $c a .25 \%$ ionic liquid was leached after six runs. 

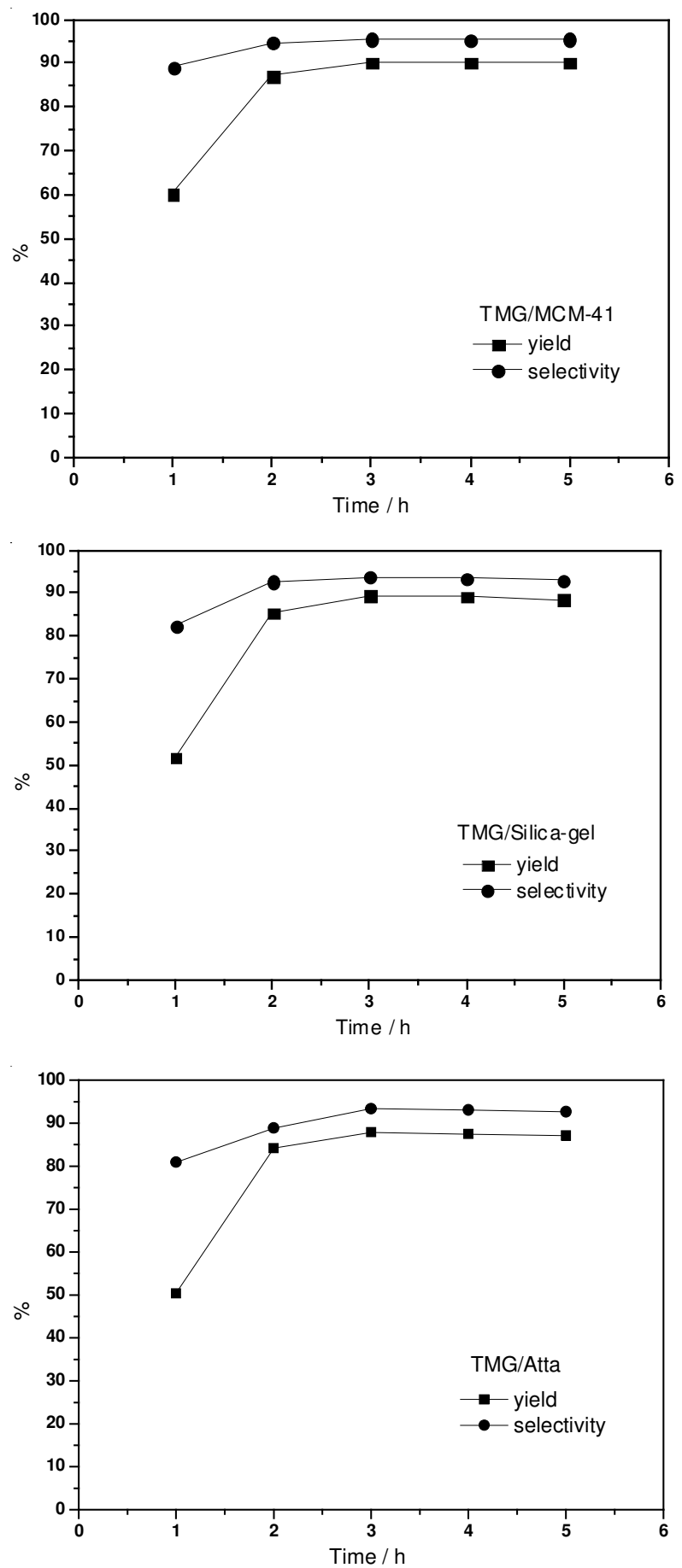

Fig. 7. Effect of reaction time on the yield of propylene glycol methyl ether and the isomer selectivity. Reaction conditions: PO, $0.1 \mathrm{M}$; $\mathrm{MeOH} / \mathrm{PO}$ molar ratio, 3; catalyst, $0.25 \mathrm{~g}$; reaction temperature, $353 \mathrm{~K}$

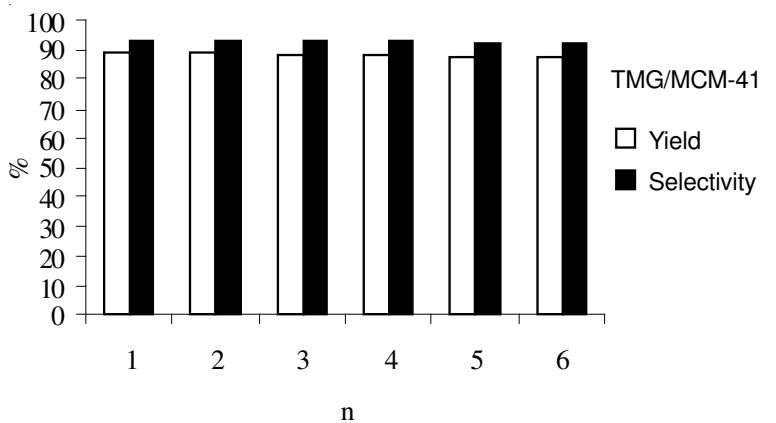

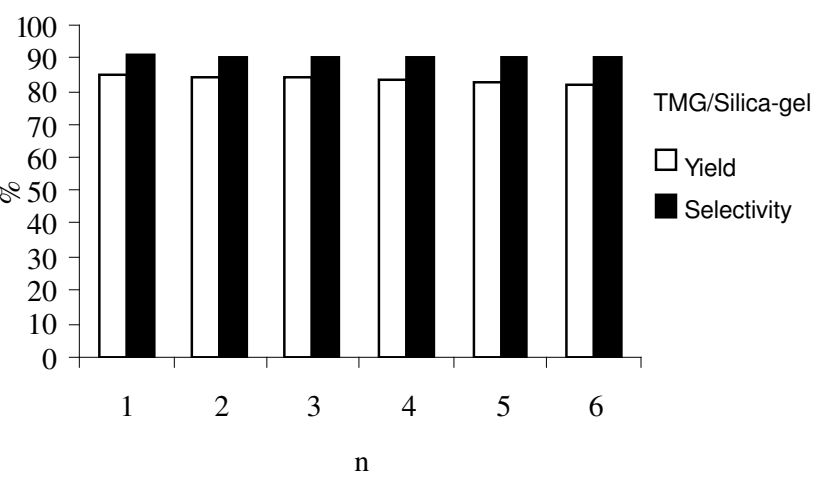

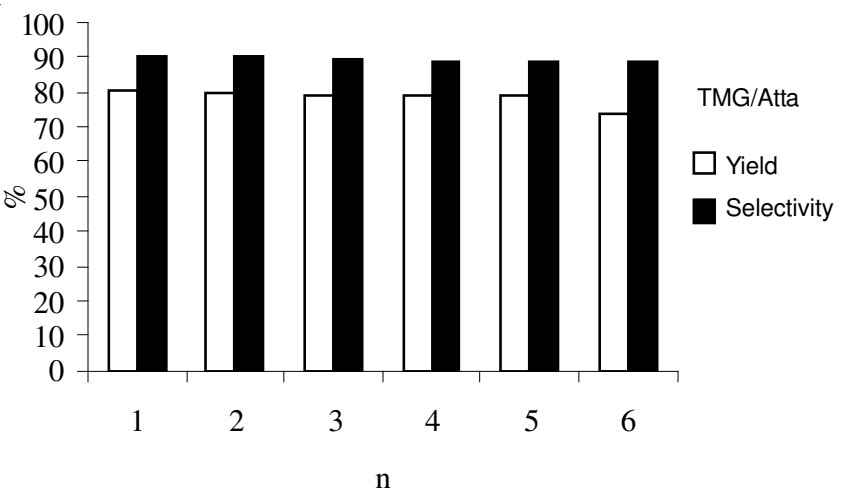

Fig. 8. Recycle of the catalyst TMG/MCM-41, TMG/Silica-gel and TMG/ Atta. Reaction conditions: PO, $0.1 \mathrm{M} ; \mathrm{MeOH} / \mathrm{PO}$ molar ratio, 3; catalyst, $0.25 \mathrm{~g}$; reaction time, $3 \mathrm{~h}$; temperature, $353 \mathrm{~K}$

\section{Conclusion}

The ionic liquid 1,1,3,3-tetramethylguanidium lactate was immobilized on three types of solid supports, MCM-41, silicagel and attapulgite, using different methods. The materials obtained were proven to be excellent heterogeneous catalysts for the reaction of methanol with propylene oxide to produce propylene glycol methyl ether. 1-Methoxy-2-propanol was the predominant product. At the optimized reaction conditions, the yield of propylene glycol methyl ether and the selectivity to 1-methoxy-2-propanol over TMG/MCM-41 are as high as 89 and $93 \%$, respectively. TMG/MCM-41 has a higher catalytic activity than TMG/Silica-gel and TMG/Atta. The three catalysts can be recovered easily and reused for at least six cycles without decrease in activity and selectivity.

The resulting catalyst is active and stable for synthesizing propylene glycol methyl ether. The catalysts not only combine the advantages of the high surface area materials and ionic liquid, but also show synergetic effects for the reaction. This green and effective method can be used to prepare some other catalysts via immobilization of ionic liquids on support materials.

\section{ACKNOWLEDGEMENTS}

This work was supported by the Shanghai Municipal Natural Science Key Research Project J51503 of China and Shanghai Municipal Natural Science Foundation 11YZ 221 of China. 


\section{REFERENCES}

1. P. Wasserscheid and T. Welton, Wiley-VCH, Weinheim (2008).

2. V. Calo, A. Nacci, A. Monopoli and P. Cotugno, Chem. Eur. J., 15, 1272 (2009).

3. N. Yan, Y. Yuan, R. Dykeman and P.J. Dyson, Angew. Chem., 49, 5549 (2010).

4. R. Sheldon, Chem. Commun., 2399 (2001).

5. J. Dupont, R.F. de Souza and P.A.Z. Auarez, Chem. Rev., 102, 3667 (2002).

6. W.A. Herrmann, Angew. Chem., 41, 1290 (2002).

7. C.P. Mehnert, N.C. Dispenziere and R.A. Cook, Chem. Commun., 1610 (2002).

8. Y.R. Jorapur and D.Y. Chi, Bull. Korean Chem. Soc., 32, 3130 (2011).

9. I.M. Pastor and M. Yus, Curr. Org. Chem., 9, 1 (2005).

10. C.E. Song, C.R. Oh, E.J. Roh and D.J. Choo, Chem. Commun., 1743 (2000).
11. D.B. Zhao, Z.F. Fei, T.J. Geldbach, R. Scopelliti and P.J. Dyson, J. Am. Chem. Soc., 126, 15876 (2004).

12. H. Gao, B. Han, J. Li, T. Jiang, Z. Liu, W. Wu, Y. Chang and J. Zhang, Synth. Commun., 34, 3083 (2004).

13. Y.H. Wang, L.Q. Yuan, L. Yu and T.T. Xiang, CIESC J., 61, 2565 (2010).

14. Z.Q. Wang, Y.M. Zhou, Y.Q. Sun, K. Fan, X.X. Guo and X.L. Jiang, J. Solid State Chem., 182, 2130 (2009).

15. R.E. Parker and N.S. Isaacs, Chem. Rev., 59, 737 (1959).

16. P. A. Martin, T. William and C. Malama, US Patent, 5,110,992 (1992).

17. W.J. Smith and F.C. Malherbe, US Patent, 6,291,720B1 (2001).

18. S.G. Liu, X.L. Zhang, J.P. Li, N. Zhao, W. Wei and Y.H. Sun, Catal. Commun., 9, 1527 (2008)

19. W.Y. Zhang, H. Wang, W. Wei and Y.H. Sun, J. Mol. Catal. A, 231, 83 (2005).

20. W.Y. Zhang, H. Wang, Q.B. Li, Q.N. Dong, N. Zhao, W. Wei and Y.H. Sun, Appl. Catal. A, 294, 188 (2005). 